\title{
Machine Flow based Energy-Power Approximation on Elastic Cloud Services
}

\author{
A. Ramesh Kumar \\ Research Scholar \\ Department of Computer Science, \\ Manonmaniam Sundaranar University
}

\author{
C. Chandrasekar, PhD \\ Professor \\ Department of Computer Science, \\ Periyar University.
}

\begin{abstract}
Cloud computing offers cloud mobile services with different elasticity application. The role of elasticity in cloud mobile services offers an effective mapping application on the cloud zone that matches with the resources that can be allocated with which it actually requires. Huge number of techniques have proposed in the past using energy-aware resource allocation that used heuristics method and offered with substantial amount of cloud services but failed to enhance the energy efficient management on elastic cloud computing environments. In addition to secure the Mobile Cloud Computing (MCC) guarantees the user privacy but failed to include a host trusted domain module for other cloud service providers. To attain an energy efficient system for the cloud mobile services, a method called Machine Flow based Energy-Power Approximation (MFEPA) is presented in this paper. The method MFEPA is executed for each elastic cloud services for efficient energy-power saving in the cloud mobile devices. Moreover with the design of MFEPA algorithm two objectives are attained. At first with the application of Multigrid approximation technique, the energy consumption is reduced. Next, in order to reduce the power consumption for mobile cloud services, a look-ahead control is introduced. Multiple grid (i.e.,) machines is reduced to a coarser construction, and the solution is mapped back to the inventive grid. The mapping reduces the energy during the unnecessary computing load and proves to be effective on the terminal mainframe mobile communications. The Look-ahead control in MFEPA assigns weights to all the users and decreases the power usage on the wireless interface. The power minimized up to $9.42 \%$ averagely on different experimental results and reduces the performance degradation on the elasticity of cloud applications. MFEPA method performs the experimental work on the factors such as true positive rate, energy efficiency level, and grid mapping efficacy rate.
\end{abstract}

\section{Keywords}

Cloud mobile devices, Machine Flow based Energy-Power Approximation, Look-ahead Control, Multiple Grid, and Coarser Structure.

\section{INTRODUCTION}

Cloud computing has been extensively applied for the future generation of computing infrastructure. Cloud mobile services assure flexible services for different ranges of business customer requirements which are isolated in the form of infrastructure. The cloud mobile computing policy defines the algorithms on different QoS expectations such as energy, power, CPU load, memory and communication cost. With the increasing business operations started using Internet, the elastic application is not effective for the transparent use of cloud mobile resources.
The business operation includes multiple component and the components are rearranged based on the workload of business logic. The business logic with elasticity applications execute parallel and drop-in replacing with the amount of intelligence obtained from the business environments. Moreover with the complexity of cloud mobile model, the energy is not effective on saving large scale real world systems.

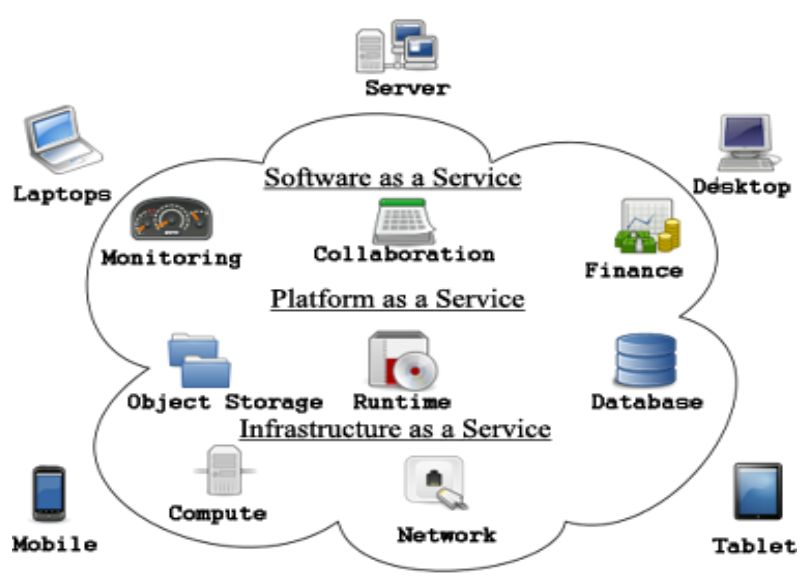

Figure.1: Cloud Business Service Structure

Figure 1 describes the model for business structure involving cloud services. Mobile devices are more and more becoming a significant part of human life. Mobile devices are also proved to be most effectual but at the same time appropriate communication tools are not with this point and position. Mobile users construct wider knowledge from the variety of services from mobile applications. The mobile application runs on different types of devices via wireless networks. The fast development of mobile computing (MC) becomes an influential trend in the development of IT technology. Though, the mobile devices are extensively used, numerous challenging works need to be carried out in the cloud infrastructure.

SLA in cloud computing is definite upon a business process as it provide end to-end Quality of Service (QoS) constraint. This is because a process defines abstract services and interrelates with an accomplished and definite goal. Mobile resource workload executed in cloud environment [20] was not proved to be effective in succeeding the probabilistic analysis result. An approach for data placement policy named Matrix based k-means clustering in [6] was used to cluster the data in order to appropriate with the data centers. But matrix based k-means clustering consumed more execution time on the cloud workflows of mobile services. Rich Internet Applications (RIA) in the mobile cloud zone as illustrated in [4] was though proved to be practical and correct on several computational mobile environment tasks, it cannot perform 
lesser power in case of excessive computing load and inefficient on the terminal mainframe communications. Ontology based approach as demonstrated in [19] examined cloud service compatibility by applying reasoning on the authority knowledge. But with the application of fuzzy Preferences, it semantically failed to enrich the new formalisms to further enhance the translator module in cloud mobile services. In order to predict the execution time, a framework to support knowledge discovery in cloud environment was presented in [21]. To provide the computation resources in dynamic manner, the work in [21] included an elastic algorithm based on Poisson distribution to learn and make customize diagnosis to ensure the quality of service.

Data replication model in [7] implemented after the request redirection using the cloud service provisioning on data grid environment. The repository takes the logical filename and the associative characteristics to easily choose the optimal replica site from mobile services but consumed more power while performing the replica operation. Enhanced Dynamic Hierarchical Replication (EDHR) method as demonstrated in [9] obtained the maximum benefit from replication without any maximal power consumption. EDHR method with QoS factors based fault tolerance was not considered with dynamic replication in cloud zone.

Dynamic Voltage Frequency Scaling as illustrated in [15] performed fine grained variation in identifying the faults using survive migration. With the introduction of migration, it minimized the involvement of active physical nodes but the virtual machine was not proved to be active in the system. Dynamic Request Redirection and Elastic Service Scaling in [11] were implemented using the diverse allocation strategy in mobile cloud to attain the robustness. But Dynamic Request Redirection failed to develop secure distributed cloud provisioning system on the grid zone files. Secure mobile cloud computing as described in [2] adopted services to the risk platforms. Mobile Cloud Computing (MCC) guaranteed the user privacy but failed to have a host trusted domain module on other cloud service providers.

Collaborative provable data possession scheme in cloud environment accept homomorphic verifiable reaction techniques in [12] to perform the verification process. The homomorphic verifiable responses also integrate the hash index hierarchy with the challenging problem. Data storage in cloud as shown in [13] utilized public key based homomorphic authenticator with accidental masking privacy preserving but the data were not accessed with higher bandwidth rate.

The stored file patterns as carried out in [5] was accessed and perform the file integration on the Hadoop Distributed File System (HDFS) to achieve higher bandwidth rate. The grid files integrated with the theoretical formulation provided the result with the help of merging operation but faults in cloud service provisioning was not examined. Energy efficient locating sensing methods as described in [17] are accept to get hold of correct mobile location information by spending minimal energy.

Energy-Efficient locating sensing method as demonstrated in [3] provided better understanding on design principles and challenges in the mobile cloud computing but energy was not efficiently used on the large dataset. The energy-aware resource allocation in [1] used heuristics method by offering significant cloud services for both the resource providers and consumers. But the QoS and power usage characteristics of the devices failed to improve the energy-efficient organization on elastic cloud computing environments.

In this work, focus is made on developing a Machine Flow based Energy-Power Approximation (MFEPA) Algorithm. MFEPA is constructed with the aim of reducing the power of the cloud mobile devices. The initial work involved in MFEPA is to reduce the energy consumption using the Multigrid approximation technique. Second part in MFEPA is to reduce the power consumption on the mobile cloud services using a look-ahead control. With this the MFEPA algorithm reduces the memory consumption, so that the profit margin of the business providers with Infrastructure as a Service (IaaS) is also increased.The formation of this paper is as follows. In Section 1, describes the basic problems in energy maintenance on large dataset. In Section 2, an overall view of the Machine Flow based Energy-Power Approximation (MFEPA) is presented. Section 3 and 4 outline experiment results with parametric factors and present the result graph for research on mobile cloud environment. As a final point, Section 5 demonstrates the related work and Section 6 concludes the work with better result outcome.

\section{MACHINE FLOW BASED ENERGY - POWER APPROXIMATION ON ELASTIC CLOUD SERVICES}

The goal of MFEPA method is to provide energy-power saving mobile services on elastic cloud services. MFEPA method includes two phases. The first phase involved in the design of MFEPA is to develop a Multi-grid approximation technique to reduce the energy consumption level on cloud mobile services. Multi-grid approximation takes into consideration the workload of each mobile service within a specific time period to approximate the resources on mobile services. Multi-grid approximation technique obtains the solution with minimal energy consumption by replacing the position of mobile devices.

The second phase involved in the design of MFEPA method is to implement the look-ahead control in order to reduce the power consumption on mobile cloud services. The look-ahead control in MFEPA method uses the weighting factor to all the mobile cloud users for reducing the power consumption and maximizes the profit margin rate. The architecture Diagram of Machine Flow based Energy-Power Approximation is described in Figure 2 


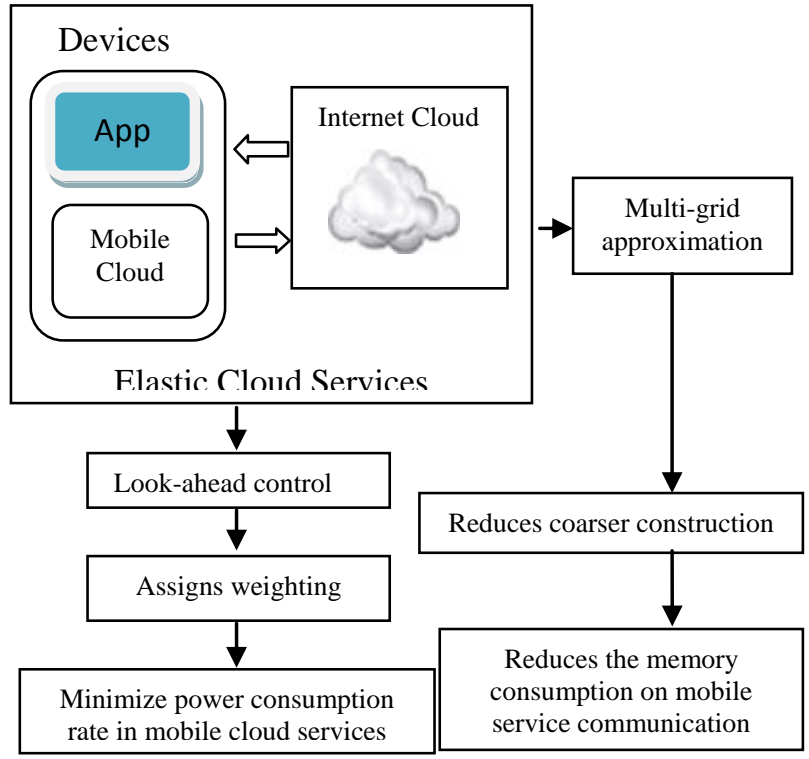

Figure 2: Architecture Diagram of MFEPA method

Figure 2 illustrates the architecture diagram of MFEPA method where the mobile applications are placed in the internet cloud. In MFEPA method, the elastic cloud services include the mobile cloud devices and internet cloud applications where the mobile application is accessed from the cloud with effective resource management. MFEPA method uses the multi-grid approximation method to reduce the coarser construction where the multi-grid controls the time period continuously to optimize the system performance with the minimal energy consumption. The power consumption in MFEPA method for elastic cloud services is also reduced using the Look-ahead control.

The workload of the mobile services in MFEPA method changes quickly which means that the workload changes on the basis of time period with the application of look-ahead control that makes high performance with optimized power control. Multi-grid approximation is combined with the look-ahead control to attain effective machine flow with minimal power and energy consumption. The reduction of the energy and power rate attains higher profit margin on the Infrastructure as a Service (IaaS) based business providers. The details of each phases is described elaborately in the following sections.

\subsection{Multi-grid Approximation Technique}

The multi-grid approximation technique in MFEPA considers the workload of each mobile service within the specific interval in order to perform resource approximation on mobile services. In MFEPA method, let us consider the set of mobile services with ' $n$ ' active cloud servers in the internet cloud zone. The mobile services in the internet cloud zone allocate the resources based on the request count with the request count denoted in matrix set. In MFEPA method, let the matrix $M_{i, j}$ denotes the resources allocated from services ' $i$ ' to server ' $j$ '. Here the service ' $i$ ' denotes the service request path whereas ' $j$ ' denotes the server path which accept the request points.

MFEPA method uses the matrix set with multiple grids to reduce the coarser construction and to define the resource capacity of each server. The resources required for mobile services ' $i$ ' for the time interval $T_{i}$ considers the basic resource used for efficient energy-power saving in the cloud mobile devices. The resource requirement of mobile services in MFEPA method satisfies the resource allocation on the internet cloud zone.

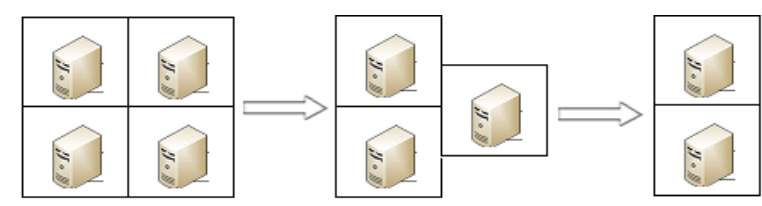

Figure 3: Multiple Grid Reduction Structure in Cloud

Figure 3 demonstrates the multiple grid reduction structure in the internet cloud zone. The grid thus reduced to a coarser construction minimizes the energy consumption rate. Multigrid approximation using MFEPA method for ' $n$ ' resources $\mathrm{R}_{1}, \mathrm{R}_{2}, \mathrm{R}_{3}, \ldots, \mathrm{R}_{\mathrm{n}}$ formulates the mathematical as given below.

Energy level $=\min \sum E\left(\mathrm{R}_{1}, \mathrm{R}_{2}, \mathrm{R}_{3}, \ldots, \mathrm{R}_{\mathrm{n}}\right)$

The energy level of every resource is analyzed using MFEPA method, which produces reasonable result to reduce the grid structure. The resource management in MFEPA method dynamically adopts the scale with easy mapping of the original grid in the cloud infrastructure. MFEPA method considers CPU to easily extent the multi dimension resources and reduces the energy for excessive computing load. The easier way of terminal mainframe mobile communications is carried out using MFEPA method with the multiple grid reduction where the values of $i$ and $j$ ranges from $1,2,3, \ldots, \mathrm{n}$ in MFEPA method is demonstrated as,

Grid Reduction Form $=\min \sum \sum\left|\mathrm{M}_{\mathrm{i}, \mathrm{j}}-\mathrm{M}_{\mathrm{i}, \mathrm{j}}^{*}\right|$

Here in (2), $\mathrm{M}_{\mathrm{i}, \mathrm{j}}^{*}$ denotes the mobile services of the current grid where $\mathrm{M}_{\mathrm{i}, \mathrm{j}}$ represents the older matrix grid and the solution is mapped with the $\mathrm{M}_{\mathrm{i}, \mathrm{j}}^{*}$ grid. The major goal of MFEPA method is achieved using the multi-grid approximation that reduces the energy consumption in the cloud infrastructure. The four simple steps carried out in the design of multi-grid approximation technique is described as,

Step 1: Continuously collects information from cloud mobile services

Step 1.1: Contains mobile service workload, QoS and Resource energy Utilization level

Step 2: Predict workload on each mobile service with $T_{i}$ interval

Step 2.1: Predicted workload placed in the matrix grid

Step 3: Analyze matrix grid and compute the grid to reduce the matrix (i.e.,) grid form from multiple grid structure

Step 4: Executes the cloud resources by satisfying mobile services and minimal energy consumption

The first objective in MFEPA method denotes minimization of energy consumption by avoiding too much of mapping time in cloud internet zone. Multiple grid (i.e.,) machines is reduced to a coarser construction, and the solution is mapped back to the original grid. With this, the mapping reduces the energy on the excessive computing load with effective communication on mobile services. Finally, resource allocating solution is identified which minimizes the total energy using the multiple grid form. 


\subsection{Look-ahead control Mechanism}

The second objective of MFEPA method is to reduce the power consumption on the grid connections where the power of the system is managed using the look-ahead control. The advantage of using the look-ahead control is to take a correct decision with the weighting factor that in turn decreases the power usage on the cloud zone. With this the workload in the cloud zone with the mobile services changes rapidly using MFEPA method, that easily cover the entire user request with improved true positive ratio. The weighting factor in look-ahead control helps to easily control the excessive switching of resources using the mobile services as the weighting factor monitors the workload variability as well as the power consumption rate.

The weighting factor in look-ahead control estimates the relative importance of each users request and groups similar requests. Similar requests contain the same weighting factor as a result the waiting time for the resource utilizations is minimized. With this the minimization of waiting time reduces the power consumption and the weighting factors is reviewed periodically in the look-ahead control and adjusted based on the user needs. Look-ahead control measures the weighting factor based on the resource utilization level, and amount of user request to the same mobile application services as given below.

Weighted Factor $=$ RUL weight + Request count

The weighting factor of 'RUL' denotes the Resource utilization level on the IaaS point. The higher weighting factor value is considered to be the top priority level, and top priority level task is provided to utilize the mobile services. Finally, MFEPA method provides the obtained first result to the top priority users and determines an accurate overall performance rating. Look-ahead control based power model in MFEPA method is formularized as,

Weighting Factor $\mathrm{P}(\mathrm{u})=\mathrm{K} * \mathrm{P}_{\max }+(1-\mathrm{K}) * \mathrm{P}_{\max } * \mathrm{u}$

The parameter Weighting factor $\mathrm{P}(\mathrm{u})$ denotes the current power consumption with the weighting factor in MFEPA method and $\mathrm{P}_{\max }$ denotes the maximum power consumed on the operation for the resource consumption, ' $u$ ' on the mobile services where ' $k$ ' is the portion of power consumed by server in cloud computing.

\subsection{Combining Energy and Power Aware System in MFEPA method}

The integration of energy and power easily identify the profit margin of the cloud users while using the Infrastructure as a Service (IaaS). The timing period of MFEPA method defines the model with power and energy consumption rate with an integral factor ranging between $\mathrm{t} 1$ and $\mathrm{t} 2$ as,

$$
\text { Timing period }=\int \text { Weighting Factor } \mathrm{P}((\mathrm{u}) \mathrm{t}) * \mathrm{dt}
$$

The weighting factor is defined as the time period with which the mobile services vary from the time position ' $t 1$ ' to ' $\mathrm{t} 2$ ' and $\mathrm{P}(\mathrm{u}) \mathrm{t})$ denotes the unit of power consumed for particular mobile services on the cloud zone. The algorithmic step of the MFEPA method is described as,

\section{// Machine Flow based Energy-Power Approximation Algorithm}

Input: Set of servers ' $S$ ', Time Interval $T_{i}$, Resources ' $R$ '
Output: Energy and power aware mobile services to cloud infrastructure

Step 1: Select the set of servers which perform the mobile services on cloud zone

Step 2: While true

Step 3 : Select the resources for $\mathrm{R}_{1}, \mathrm{R}_{2}, \mathrm{R}_{3}, \ldots, \mathrm{R}_{\mathrm{n}}$

Step 4 : If satisfy the user request, then

Step 5: Computes min $\min \sum \sum\left|\mathrm{Mi}, \mathrm{j}-\mathrm{M}^{*} \mathrm{i}, \mathrm{j}\right|$ with $\mathrm{i}$ and $\mathrm{j}$ values ranging between 1 and $\mathrm{n}$ for reducing the grid forms

Step 6: Multi-grid Approximation steps from Figure 4 calculate energy rate

Step 7: Optimize the solution based on the minimal energy rate

\section{Step 8: End if}

Step 9: Look-ahead control cover the entire user request with weighting factors

Step 10: Weighting Factor $\mathrm{P}(\mathrm{u})=\mathrm{K} * \mathrm{P}_{\max }+(1-\mathrm{K}) * \mathrm{P}_{\max }$ $* \mathrm{u}$ provides the obtained first result to the top priority users.

Step 11: Time period of the each cloud users defines the energy and power aware mobile service system

\section{Step 12: End While}

The algorithm selects the 'S1', 'S2', up to 'Sn' cloud server to perform resource allocation on mobile services. The server accepts the service requests, and uses the multi-grid approximation technique to reduce the grid points and perform effective mapping in sensor network. The reduction of grip points reduces the energy consumption in cloud infrastructure. The look-ahead control with the weighting factor reduces the power consumption rate at different time interval.

\section{EXPERIMENTAL EVALUATION OF MFEPA ALGORITHM}

This section demonstrate the effective of Machine Flow based Energy-Power Approximation (MFEPA) algorithm with the experimental evaluation performed in JAVA platform using CloudSim simulator using statlogheart dataset extracted from UCI. The particular toolkit is chosen as a simulation platform as it is a present simulation structure in Cloud environments. Experimental machine is simulated with cloud data center comprising of 4 GB RAM of storage in client machines. The user's current needs for effective communication provisioned assorted Virtual Machine (VM) pack. Each VM runs a web-application with variable business based query request from client, which is modeled to generate the utilization of bandwidth according to consistently distributed random variable.

The goal of CloudSim is to provide a generalized and extensible simulation framework on the cloud data center with the server and client mobile service systems that enables to model, simulate, and experiment on MFEPA algorithm. The MFEPA algorithm is compared against the existing Energy-aware Resource Allocation Heuristics (ERAH) method and Secure Mobile Cloud Computing (SMCC) system. True positive rate, Energy efficiency level, Grid mapping efficacy rate, power consumption rate, profit margin rate and grid mapping time are the experimental factors taken for the result analysis. 
The table1 is evaluating the performance of true positive rate using the MFEPA algorithm. The cloud users used in this experiment ranges from 5 to 35 cloud users. The performance of true positive rate is measured in terms of percentage that defines the ability to test the mobile services depending on the users need. The test result with the high positive rate provides higher sensitivity rate. The true positive rate is defined as

True Positive Rate $=[(\mathrm{TPMS}) /(\mathrm{TP}+\mathrm{FN}) * 100]$

Where TPM, TP, FN represent the number of true positive mobile services and results of true positive and false negative respectively. In order to minimize the energy consumption rate, a multi-grid approximation technique is presented that allocates the resources based on the request count denoted by matrix set. In the experimental setup, the mobile services considered ranges from 20 to 140 . The results of 7 different request placed by the mobile services are listed in table 2.

As listed in table 2, MFEPA measures the amount of energy consumed while providing mobile services to cloud environment which is measured in terms of Joules $(\mathrm{J})$. The energy consumed using the method MFEPA offer comparable consumption values than the state-of-the-art methods. The table 3 is further compare the mapping of coarser construction of the proposed method using the MFEPA algorithm. The experiments were conducted using the grid count in the range of 50 to 350 that measures the grid mapping efficiency rate which is measured in terms of percentage $(\%)$.

The table 4 is show the analysis of power consumption with respect to service count ranging between 20 and 140 that measures the amount of power consumed to perform the mobile services on the cloud users measured in terms of Kilowatt (kW). The power consumption is defined as

Power Consumption $=$ Number of mobile services $*$ current flow (in terms of volt)

In table 5 the profit margin rate in MFEPA is evaluated for seven iterations that measures the profitable rate attained while using the IaaS information from the cloud zone which varies as per the user resources attained from the cloud zone. The profit margin rate is defined in terms as the percentage (\%). Finally, table 6 provides the processing (grid) mapping time with varying grid size of 10 to $70 \mathrm{~KB}$ that measures the amount of time consumed to perform the operation in the mobile services. The grid mapping time is evaluated using the difference between the starting time to map the grid and ending time after mapping the grid with the mapping time measured in terms of milliseconds (ms).

Processing (i.e., mapping) Time $=\mathrm{PT}_{1}-\mathrm{PT}_{2}$

\section{RESULT ANALYSIS}

To better understand the effectiveness of the proposed MFEPA method, extensive experimental results are reported in table 1. The MFEPA algorithm is compared against the existing Energy-aware Resource Allocation Heuristics (ERAH) method and Secure Mobile Cloud Computing (SMCC) system. Cloudsim simulator is used to experiment the factors and analyze the result percentage through table and graph values. Results are presented for different number of cloud users considering the true positive and false negative values. The results reported here confirms that with the increase in the number of cloud users, as the number of true positive value increases the true positive rate also increases. Finally, the value of true positive rate gets saturated when the number of cloud users ranges from 2035.

Table 1 Tabulation of True Positive Rate

\begin{tabular}{|c|c|c|c|}
\hline \multirow{2}{*}{$\begin{array}{c}\text { No. of } \\
\text { Cloud } \\
\text { users }\end{array}$} & \multicolumn{3}{|c|}{ True Positive Rate (\% ) } \\
\cline { 2 - 4 } & $\begin{array}{c}\text { ERAH } \\
\text { method }\end{array}$ & $\begin{array}{c}\text { SMCC } \\
\text { system }\end{array}$ & $\begin{array}{c}\text { MFEPA } \\
\text { Algorithm }\end{array}$ \\
\hline 5 & 85 & 90 & 96 \\
\hline 10 & 80 & 87 & 95 \\
\hline 15 & 80 & 89 & 96 \\
\hline 20 & 82 & 90 & 95 \\
\hline 25 & 83 & 91 & 97 \\
\hline 30 & 84 & 91 & 98 \\
\hline 35 & 85 & 92 & 98 \\
\hline
\end{tabular}

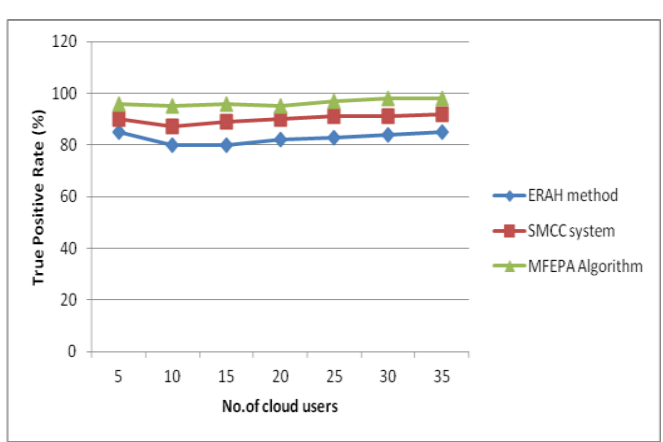

Figure 4: Performance of True Positive Rate

Figure 4 illustrate the true positive rate based on the cloud users. The proposed MFEPA algorithm performs relatively well when compared to two other methods ERAH method [1] and SMCC system [2]. The algorithm had better changes where the workload in the cloud zone with mobile services changes rapidly that helps to easily cover the entire user request with improved true positive rate using the weighting factor. MFEPA method easily covers the entire user request with improved true positive rate by $12-20 \%$ when compared with the ERAH method [1]. The weighting factor monitors the workload variability based on the resource utilization level, and amount of user request and improves the true positive rate by $5-9 \%$ when compared with the SMCC system [2].

Table 2 Tabulation of Energy Consumption rate

\begin{tabular}{|c|c|c|c|}
\hline \multirow{2}{*}{$\begin{array}{c}\text { No. of } \\
\text { mobile } \\
\text { services }\end{array}$} & \multicolumn{3}{|c|}{ Energy Consumption rate (J) } \\
\cline { 2 - 4 } & $\begin{array}{c}\text { ERAH } \\
\text { method }\end{array}$ & $\begin{array}{c}\text { SMCC } \\
\text { system }\end{array}$ & $\begin{array}{c}\text { MFEPA } \\
\text { Algorithm }\end{array}$ \\
\hline 20 & 0.30 & 0.28 & 0.26 \\
\hline 40 & 0.32 & 0.29 & 0.27 \\
\hline 60 & 0.36 & 0.33 & 0.31 \\
\hline 80 & 0.37 & 0.35 & 0.33 \\
\hline 100 & 0.38 & 0.39 & 0.33 \\
\hline 120 & 0.42 & 0.40 & 0.34 \\
\hline 140 & 0.46 & 0.43 & 0.37 \\
\hline
\end{tabular}




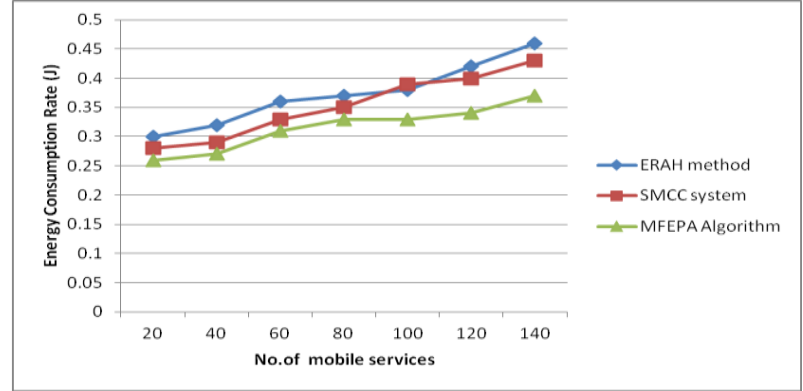

Figure 5: Measure of Energy Consumption rate

The targeting results of energy consumption rate using MFEPA with two state-of-the-art cloud services [1], [2] in figure 5 is presented for visual comparison based on the mobile services. This method differs from the ERAH method [1] and SMCC system [2] is incorporated CPU to easily extent the multi dimension resources and therefore reduce the energy consumption by providing energy efficient mechanism. For the most different number of mobile services, MFEPA achieves comparable performance to ERAH method and SMCC system. The energy consumption rate is $10-19 \%$ better when compared with the ERAH method [1]. The terminal mainframe mobile communications is carried out in the MFEPA method with 5 - $15 \%$ lesser energy consumption when compared with the SMCC system [2].

Table 3 Tabulation of Grid Mapping Efficiency rate

\begin{tabular}{|c|c|c|c|}
\hline \multirow{2}{*}{ Grid Count } & \multicolumn{3}{|c|}{ Grid Mapping Efficiency Rate (Mapping \%) } \\
\cline { 2 - 4 } & $\begin{array}{c}\text { ERAH } \\
\text { method }\end{array}$ & $\begin{array}{c}\text { SMCC } \\
\text { system }\end{array}$ & $\begin{array}{c}\text { MFEPA } \\
\text { Algorithm }\end{array}$ \\
\hline 50 & 75 & 78 & 80 \\
\hline 100 & 73 & 76 & 82 \\
\hline 150 & 75 & 79 & 84 \\
\hline 200 & 77 & 80 & 85 \\
\hline 250 & 78 & 82 & 87 \\
\hline 300 & 79 & 83 & 89 \\
\hline 350 & 81 & 86 & 91 \\
\hline
\end{tabular}

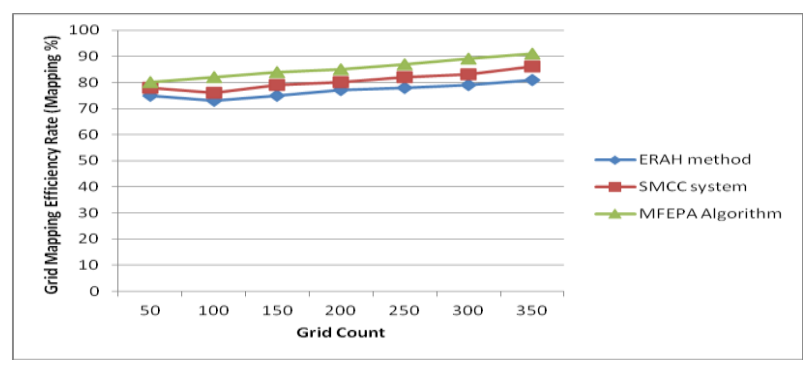

Figure 6 : Performance comparison of the proposed solution versus the ERAH method [1] and SMCC system[2] with respect to Grid Mapping Efficiency rate

Figure 6 presents the variation of grid mapping efficiency rate of MFEPA algorithm over different number of grid count. All the results provided in figure 7 establish that the proposed MFEPA algorithm significantly outperforms the other two methods, ERAH method [1] and SMCC system [2]. The better performance of MFEPA algorithm is achieved due to the fact that it provides an efficient way to provide multi-grid approximation for different grid count values. As a result, the MFEPA method produces the reasonable result to reduce the grid structure. The reduction of grid structure, improves the efficiency rate by $6-12 \%$ when compared with ERAH method [1]. MFEPA method dynamically adopts the scale with the easy mapping of the original grid in the cloud infrastructure by improving the mapping efficiency rate by $2-7 \%$ when compared with the SMCC system [2].

Table 4 Tabulation of Power Consumption Rate

\begin{tabular}{|c|c|c|c|}
\hline \multirow{2}{*}{$\begin{array}{c}\text { Service } \\
\text { Count }\end{array}$} & \multicolumn{3}{|c|}{ Power Consumption Rate (kW) } \\
\cline { 2 - 4 } & $\begin{array}{c}\text { ERAH } \\
\text { method }\end{array}$ & $\begin{array}{c}\text { SMCC } \\
\text { system }\end{array}$ & $\begin{array}{c}\text { MFEPA } \\
\text { Algorithm }\end{array}$ \\
\hline 20 & 250 & 220 & 200 \\
\hline 40 & 285 & 265 & 240 \\
\hline 60 & 420 & 390 & 360 \\
\hline 80 & 475 & 450 & 420 \\
\hline 100 & 555 & 525 & 500 \\
\hline 120 & 685 & 650 & 620 \\
\hline 140 & 900 & 870 & 840 \\
\hline
\end{tabular}

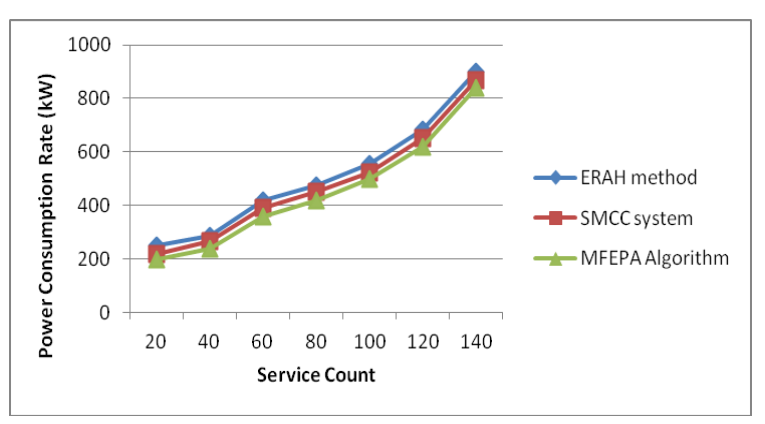

Figure 7: Power Consumption Rate Measure

To explore the influence of rate of power consumption on MFEPA algorithm, the experiments were performed by varying the service counts as shown in figure 7 . It also shows that the MFEPA algorithm shows competitive results with the state-of-the-art methods, namely ERAH method [1] and SMCC system [2]. Even though MFEPA algorithm uses energy-power approximation, low power consumption was recorded especially at service counts 20 and 40. This is because the MFEPA method manages the power using the look-ahead control. The power consumption is reduced by 6 $-20 \%$ when compared with the ERAH method. The lookahead control is used to take a correct decision with the weighting factor that decreases the power usage on the cloud zone by $3-9 \%$ when compared with the SMCC system.

Table 5 Profit Margin Rate Tabulation

\begin{tabular}{|c|c|c|c|}
\hline \multirow{2}{*}{ Iterations } & \multicolumn{3}{|c|}{ Profit Margin Rate (\%) } \\
\cline { 2 - 4 } & $\begin{array}{c}\text { ERAH } \\
\text { method }\end{array}$ & $\begin{array}{c}\text { SMCC } \\
\text { system }\end{array}$ & $\begin{array}{c}\text { MFEPA } \\
\text { Algorithm }\end{array}$ \\
\hline 1 & 73 & 82 & 86 \\
\hline 2 & 74 & 84 & 87 \\
\hline 3 & 74 & 87 & 91 \\
\hline
\end{tabular}




\begin{tabular}{|c|c|c|c|}
\hline 4 & 78 & 88 & 92 \\
\hline 5 & 79 & 90 & 93 \\
\hline 6 & 82 & 90 & 95 \\
\hline 7 & 83 & 91 & 96 \\
\hline 8 & 83 & 92 & 97 \\
\hline
\end{tabular}

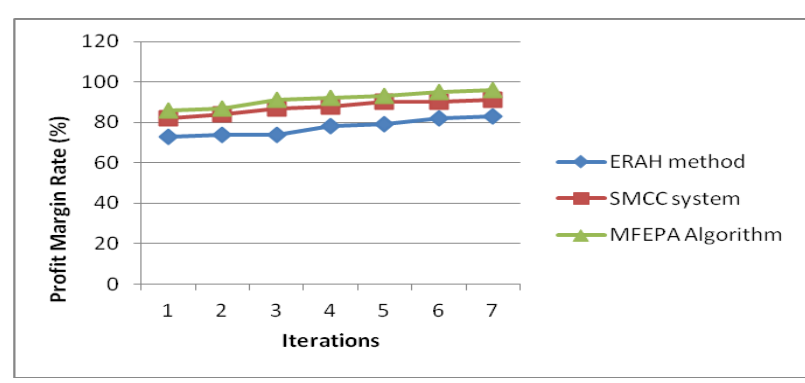

Figure 8: Profit Margin Rate Measure

To verify the performance of MFEPA algorithm more deeply, profit margin rate as illustrated in figure 8 is presented based on different iteration count. From the figure it is illustrative that the proposed MFEAPA algorithm potentially yield significant results compared to other stateof-the-art methods. The better performance of the proposed method is achieved due to the fact the MFEPA algorithm combines the energy and power factor through multi-grid approximation technique. This in turn helps to increase the profit margin rate of the cloud users by $15-22 \%$ when compared with the ERAH method [1]. Also with the introduction of the weighting factor that defined the time period with which the mobile services varied from the time positions, the profit margin rate gets improved aby $3-5 \%$ when compared with the SMCC system [2].

Table 6 Tabulation for Grid Mapping Time

\begin{tabular}{|c|c|c|c|}
\hline \multirow{2}{*}{$\begin{array}{l}\text { Grid Size } \\
\text { KB) }\end{array}$} & \multicolumn{3}{|c|}{ Processing (Grid) Mapping Time (sec) } \\
\cline { 2 - 4 } & $\begin{array}{l}\text { ERAH } \\
\text { method }\end{array}$ & $\begin{array}{l}\text { SMCC } \\
\text { system }\end{array}$ & $\begin{array}{c}\text { MFEPA } \\
\text { Algorithm }\end{array}$ \\
\hline 10 & 120 & 110 & 99 \\
\hline 20 & 245 & 225 & 205 \\
\hline 30 & 362 & 332 & 312 \\
\hline 40 & 450 & 414 & 392 \\
\hline 50 & 515 & 489 & 456 \\
\hline 60 & 674 & 655 & 635 \\
\hline 70 & 775 & 750 & 717 \\
\hline
\end{tabular}

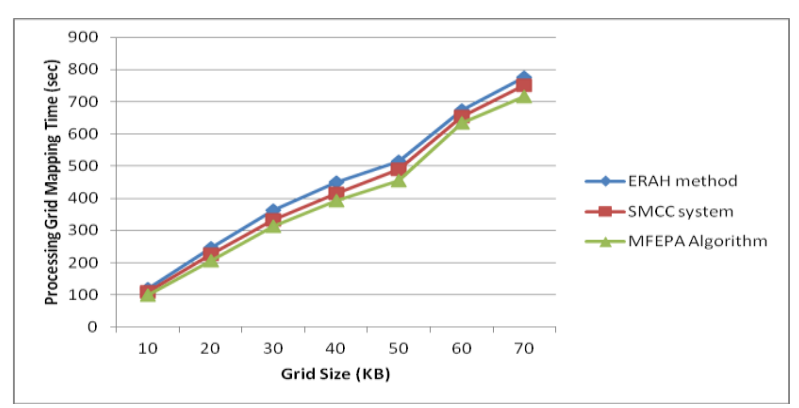

Figure 9: Grid Mapping Time Measure
Lastly the grid mapping time is measured via a grid size of $10 \mathrm{~KB}$ to $70 \mathrm{~KB}$. From the figure 9 it is illustrative that the proposed MFEPA algorithm potentially yields better results than existing ERAH method [1] and SMCC system [2]. The significant results achieved using MFEPA algorithm is because the mapping time with look-ahead control estimates the relative importance of each users request and accordingly groups similar requests. As a result, the multiple grid (i.e.,) machines is reduced to a coarser construction, and the solution is mapped back to the original grid with minimal time. The mapping time is reduced up to $5-17 \%$ when compared with the ERAH method [1], and 3-10\% reduced when compared with the SMCC system [2].

Finally, MFEPA method reduces the energy consumption using the Multi-grid approximation technique. The power minimization is carried out using the look-ahead control with weighting factor. The reduction of energy and power using MFEPA method improves the margin rate on the business using the Infrastructure as Service (IaaS) information.

\section{RELATED WORK}

Cloud Centers with High Degree of Virtualization under Batch Task Arrivals as expressed in [18] accounts for the decline of performance due to the workload at each node. The performance analysis of cloud computing incorporates a high degree of virtualization, group arrival of tasks and characteristically distributed service time.

Cloud Computing Services for Many-Tasks Scientific Computing (MTC) as demonstrated in [10] migrate and present high-quality performance on grids and parallel production infrastructures. Proto-MTC component is not carried out effectively in cloud grid zone with the maximal mobile service workload. Maximal workload on Highly Decentralized Information Accountability (HDIA) framework as illustrated in [8] provides end-to-end accountability with push and pull mode. HDIA is not expensive in providing the dynamic replication software tamper-free resistance in the cloud zone.

Agent Based Dynamic Resource Allocation (ARDA) as demonstrated in [14] provides complex procedure to improve resource allocation. Resource Brokering Agent has a provider list which holds the information about every cloud resource. But ADRA failed to implement the mobile protocol model with programming language. The mobile cloud computing (MCC) as introduced in [16] integrated cloud computing with the mobile environment and overpowers difficulty connected to the performance. But MCC failed to develop the searching process with the public cloud environment and also failed to detect the traffic lights on the house corners through a mobile device.

\section{CONCLUSION}

In this paper, Machine Flow based Energy-Power Approximation algorithm is provided for elastic cloud services. This solution avoids the computationally expensive energy and power problem on elastic cloud computing environments. As the method uses multi-grid approximation technique, the method reduces the coarser construction. As a result, the proposed MFEPA algorithm achieves comparable services reducing the energy on the unnecessary computing load and is very effective on energy-power saving. Dynamic resource provision in the cloud data center attains energy effective system using multi-grid approximation method. Look-ahead control in MFEPA method carefully analyzes the power consumption on the mobile cloud services. A 
weighting factor is used in look-ahead control and a clear result is fetched from different set of user's mobile services. A series of simulations result are performed to test the availability, efficiency and computational mapping time of the MFEPA method. The mapping time is reduced to $7.16 \%$ when compared with the SMCC method. Efficient workload distribution across cloud data centers enable the efficient grid mapping with $6.34 \%$ improved result when compared with the existing system. The combined multi-grid approximation and look-ahead control attains higher profit margin on the Infrastructure as a Service (IaaS) based business providers using MFEPA method.

\section{REFERENCES}

[1] Anton Beloglazov., Jemal Abawajy., Rajkumar Buyyaa., "Energy-aware resource allocation heuristics for efficient management of data centers for Cloud computing," Future Generation Computer Systems., Elsevier journal, 2012

[2] Abdul Nasir Khana., M.L. Mat Kiah., Samee U. Khanb., Sajjad A. Madanic., "Towards secure mobile cloud computing: A survey," Future Generation Computer Systems., Elsevier Journal, 2012

[3] Xiao Maa., Yong Cuia., Ivan Stojmenovic., "Energy Efficiency on Location Based Applications in Mobile Cloud Computing: A Survey,” Elsevier journal, 2012

[4] Saeid Abolfazlia., Zohreh Sanaeia., Abdullah Gania, Feng Xiab., Laurence T. Yang., "Rich Mobile Applications: Genesis, Taxonomy, and Open Issues," Elsevier journal, 2013

[5] Bo Dong., QinghuaZheng., FengTian., KuoMingChao., RuiMaa., RachidAnane, "An optimized approach for storing and accessing small files on cloud storage," Journal of Network and Computer Applications., Elsevier journal., 2012

[6] Dong Yuan., Yun Yang., Xiao Liu., Jinjun Chen., "A data placement strategy in scientific cloud workflows," Future Generation Computer Systems., Elsevier journal., 2010

[7] R. Kingsy Grace., R. Manimegalaib., "Dynamic replica placement and selection strategies in data grids-A comprehensive survey," Journal of Parallel Distributed Computing., Elsevier journal., 2013

[8] Smitha Sundareswaran., Anna C. Squicciarini., and Dan Lin, "Ensuring Distributed Accountability for Data Sharing in the Cloud," IEEE transactions on dependable and secure computing, vol. 9, no. 4, july/august 2012

[9] Najme Mansouria., Gholam Hosein Dastghaibyfard., "Enhanced Dynamic Hierarchical Replication and Weighted Scheduling Strategy in Data Grid," Journal Parallel Distributed Computing., Elsevier Journal., 2013

[10] Alexandru Iosup., Simon Ostermann,Nezih Yigitbasi., Radu Prodan., Thomas Fahringer., and Dick Epema., "Performance Analysis of Cloud Computing Services for Many-Tasks Scientific Computing," IEEE Tpds, Many-Task Computing, November 2010

[11] Jianhua Tang., Wee Peng Tay., and Yonggang Wen., "Dynamic Request Redirection and Elastic Service Scaling in Cloud-Centric Media Networks," IEEE Transactions on Multimedia., 2013

[12] Yan Zhu., and Shanbiao wang., "Secure Collaborative integrity verification for hybrid cloud environments," International Journal of Cooperative Information Systems Vol. 21, No. 3 165-197. DOI: 10.1142/S0218843012410018, (2012)

[13] Jachak K.B., Korde S.K., Ghorpade p.p. and Gagare g.j., "Homomorphic authentication with random masking technique ensuring privacy \& security in cloud computing, "bioinfo security informatics ISSN: 22499423 \& E-ISSN: 2249-9431, Volume 2, Issue 2, 2012, pp.-49-52.

[14] Haresh M V., Saidalavi Kaladyy., and Govindan., "Agent Based Dynamic Resource Allocation on Federated Clouds," IEEE, 978-1-4244-94774/11/\$26.00 (C2011

[15] Vrunda J. Patel., Prof. Hitesh A. Bheda., "Reducing Energy Consumption with Dvfs for Real-Time Services in Cloud Computing," IOSR Journal of Computer Engineering (IOSR-JCE)(May-Jun. 2014)

[16] Shraddha A. Jalan., Vaishali B.Bhagat., "Mobile Cloud Computing An Efficient Technique For Mobile Users,' International Journal of Computer Science and Mobile Computing., 2014

[17] [17] Xiao Maa., Yong Cuia., Ivan Stojmenovicb., "Energy Efficiency on Location Based Applications in Mobile Cloud Computing: A Survey," Elsevier journal, 2012

[18] Hamzeh Khazaei., Jelena Misic, and Vojislav B. Misic., "Performance of Cloud Centers with High Degree of Virtualization under Batch Task Arrivals," IEEE transaction on parallel and distributed systems, vol. X, no. Y, 2012

[19] Amir Vahid Dastjerdi., and Rajkumar Buyya., "Compatibility-aware Cloud Service Composition Under Fuzzy Preferences," IEEE Transactions On Cloud Computing, 2012

[20] Muhammad Abdullah Adnan., Ryo Sugihara., and Rajesh Gupta., "Energy Efficient Geographical Load Balancing via Dynamic Deferral of Workload," arXiv: 1204.2320v1 [cs.NI] 11 Apr 2012

[21] Kun Gao, Qin Wang, and Lifeng Xi," Reduct Algorithm Based Execution Times Prediction in Knowledge Discovery Cloud Computing Environment", The International Arab Journal of Information Technology, Vol. 11, No. 3, May 2014 